\title{
Interfacial Mechanism for Efficient Resistive Switching in Ruddlesden- Popper Perovskites for Non-volatile Memories
}

Authors: Ankur Solanki ${ }^{1,2}$, Antonio Guerrero ${ }^{3, *}$, Qiannan Zhang ${ }^{1}$, Juan Bisquert ${ }^{3}$ and Tze Chien Sum $^{1, *}$

Affiliations:

${ }^{1}$ Division of Physics and Applied Physics, School of Physical and Mathematical Sciences, Nanyang Technological University, 21 Nanyang Link, Singapore 637371

${ }^{2}$ Department of Science, School of Technology, Pandit Deendayal Petroleum University, Gandhinagar 382007, India.

${ }^{3}$ Institute of Advanced Materials (INAM), Universitat Jaume I, 12006 Castelló, Spain

*corresponding authors: Tzechien@,ntu.edu.sg, aguerrer@uji.es

\begin{abstract}
Ion migration, one of the causes of hysteresis effects, is the bane of halide perovskite optoelectronics. Herein, we leverage this unwelcome trait to unlock new opportunities for resistive switching using layered Ruddlesdsen-Popper perovskites (RPP) and explicate the underlying mechanisms. The ON/OFF ratio of RPP-based devices is strongly dependent on the layers and peaks at $\bar{n}=5$ demonstrating the highest ON/OFF ratio of $\sim 10^{4}$ and minimal operation voltage in $1.0 \mathrm{~mm}^{2}$ devices. A long data retention even in $60 \%$ relative humidity and stable write/erase capabilities exemplifies their potential for memory applications. Activation studies during impedance spectroscopy measurements reveal that the high resistive state is related to impeded charge injection from the contacts. A chemical reaction between migrating ions and the external contacts modify the charge transfer barrier at the interface. The interplay between perovskite compositions and external electrodes confirm that ions population, migration and their reactivity with external contacts are essential for resistive switching. Our findings uncover the origin of resistive switching and a new family of facile materials and devices for switching and memory applications.
\end{abstract}




\section{Introduction}

2019 marks the $10^{\text {th }}$ year anniversary of perovskite solar cells (PSCs) invention by Miyasaka. ${ }^{1}$ From humble beginnings of $3.8 \%$ efficiency, a whopping $24.2 \%$ to date places them in the same league as established polycrystalline $\mathrm{Si}$, and CIGS inorganic thin film solar cells. The perovskite fever has also spawned a parallel light emission field, yielding perovskite light emitting diodes (PLEDs) with external quantum efficiency $>21 \%$ in a short developmental span. ${ }^{2}$ Across both these fields, current hysteresis remains an open challenge in perovskite-based optoelectronics. ${ }^{3,4}$ In PSCs, current hysteresis lead to device instability and imprecise PCEs determination; while color purity (in addition to device instability) is a major drawback in PLEDs. ${ }^{5,6}$

Following extensive research, the possible sources of current-voltage hysteresis narrowed down to three ${ }^{7}$ : (i) charge trapping/detrapping from bulk and surface defects, (ii) migration of the excess ions due interstitial defects and (iii) ferroelectric polarization in perovskites. Specifically, ion migration across the bulk perovskite and accumulation at the interface under any external impulse like electric field or light illumination has been evidenced by different techniques. ${ }^{8,} 9$ Interfacial chemical/physical reaction gives rise to an ionic behavior distinct from the bulk. This leads to recombination losses at the interfaces, thus limiting the device performance. ${ }^{10,11}$

While current hysteresis is execration for most optoelectrical devices, it is a boon for other applications such as logic gates ${ }^{12}$, synaptic devices ${ }^{13}$, neuromorphic electronics ${ }^{14}$, data storage ${ }^{15}$, 16 and switching ${ }^{17,} 18$, where strong current hysteresis is a primary requisite. However, the mechanism to control the hysteresis is still under intense debate. Initially, the formation and rupture of the metal filaments at either electrodes are believed to be the source of current switching in perovskite-based resistive random access memory (ReRAM) devices. ${ }^{16,19,20}$ However, further studies show that the redistribution of iodine vacancies form conducting channels under an external applied field and is proposed as the main source of resistance change in perovskites. ${ }^{18,21,22}$ On the other hand, some reports suggest that the interfacial energy barrier at perovskite/electrode interface give rise to current switching. ${ }^{23}$ Recently, Ge et al. demonstrated silver iodide-induced switching in cesium-based memory devices. ${ }^{24}$ Nevertheless, the exact origin of hysteresis in perovskites remains contentious. A clear understanding of the underlying causes is essential either for mitigating hysteresis in optoelectronic devices or optimizing for resistive switching (RS) in memory application.

Herein, we leverage the hysteresis in ruddlesdsen-popper $(\mathrm{PEA})_{2}(\mathrm{MA})_{\mathrm{n}-1} \mathrm{~Pb}_{\mathrm{n}} \mathrm{I}_{3 \mathrm{n}+1}(\mathrm{PEA}=$ phenylethylammonium, MA = methylammonium) organic-inorganic hybrid perovskites for RS through tuning the structural layers and judicious selection of the external contacts. We elucidate a universal working mechanism that relies on the accumulation of ions migrated to the contacts under an applied electric field, and the subsequent chemical/physical interaction of these accumulated ions with the external contacts that control the switching behavior. Impedance spectroscopy (IS) experiments reveal the kinetics of the activation process that involves a reduction in the charge transfer resistance connected to the perovskite/contact interface through a chemical reaction. The optimal composition of $\mathrm{MA}^{+}$and $\mathrm{PEA}^{+}$molecules in the $\bar{n}=5$ sample 
yields the best RS performance with ON/OFF ratio of $\sim 10^{4}$ at 0.15 SET voltage compared to the $\bar{n}=1,3,7$ and 3D $\mathrm{MAPbI}_{3}$ samples in ITO/PEDOT:PSS/perovskite/PCBM/Ag device configuration. The $\bar{n}=5$ sample also exhibit good data retention capability and environmental stability. Surface potential and current switching in bare thin film under spectroscopic measurements rule out the metal filament formation requirement for RS. We also validate that the control on the supply of ions and the chemical/physical interactions with external contacts and thus switching behavior through the different external contacts containing $\mathrm{Ag}, \mathrm{Au}$ and $\mathrm{SnO}_{2}$.

\section{Results}

Here, we utilize different layered ruddlesdsen-popper (RP) hybrid perovskites (Fig. 1a) i.e., $(\mathrm{PEA})_{2}(\mathrm{MA})_{\mathrm{n}-1} \mathrm{~Pb}_{\mathrm{n}} \mathrm{I}_{3 \mathrm{n}+1}\left((\mathrm{PEA})_{2} \mathrm{PbI}_{4},(\bar{n}=1)(\mathrm{PEA})_{2} \mathrm{MA}_{2} \mathrm{~Pb}_{3} \mathrm{I}_{10}(\bar{n}=3),(\mathrm{PEA})_{2} \mathrm{MA}_{4} \mathrm{~Pb}_{5} \mathrm{I}_{16}(\bar{n}=5)\right.$ and $\left.(\mathrm{PEA})_{2} \mathrm{MA}_{6} \mathrm{~Pb}_{7} \mathrm{I}_{22}(\bar{n}=7)\right)$, and pure three-dimensional perovskite $\mathrm{MAPbI}_{3}(\bar{n}=\infty / 3 \mathrm{D})$ to demonstrate the excellent hysteresis properties for resistive random access memory (ReRAM) application. These layered RP perovskite films are polycrystalline in nature and $\bar{n}$ represents the dominant $n$ component for each sample as evaluated from XRD and UV-Vis absorption spectra (Fig. S1 and S2). The morphology of these perovskite films as displayed in Fig. S3 confirm the good quality of the samples with no obvious pinholes. Fig. 1b depicts the best ReRAM device configuration used for this study ITO $\backslash$ PEDOT:PSS $\backslash$ perovskite $\backslash \mathrm{PCBM} \backslash A \mathrm{Ag}$ (see SI for the details of device fabrication). ITO $\backslash$ PEDOT:PSS is the bottom electrode as a non-hysteretic electrode and silver is used as top electrode with $\sim 1.0 \mathrm{~mm}^{2}$ device area. A thin layer of PCBM $(10 \pm 2 \mathrm{~nm})$ was inserted between perovskite and Ag to minimize their direct contact which can cause electrode and perovskite degradation.

Fig. 1c shows a representative semi-log scale $I-V$ characteristics of perovskite ReRAM devices measured under DC voltage sweep $\left(0 \mathrm{~V} \rightarrow\left(\mathrm{V}_{\text {app }}\right) \mathrm{V} \rightarrow 0 \mathrm{~V} \rightarrow-\left(\mathrm{V}_{\text {app }}\right) \mathrm{V} \rightarrow 0 \mathrm{~V}\right)$ (see Fig. 3a and $\mathbf{S} 4$ for other devices). At voltages $<0.15 \mathrm{~V}$ this device is in a high resistance state (i.e., HRS or OFF state $>10^{5} \Omega$ ) with measured low current levels. Beyond a threshold voltage, the current increases with time, bringing the device to a low resistance state (i.e., LRS or ON state). This switching of the device to the LRS with a current level of $1 \mathrm{~mA}$ at higher bias is termed the "SET" process. The onset voltage $(\sim 0.15 \mathrm{~V})$ is more obvious in the log-log plot (Fig. 1d). The transition from HRS to LRS is rather sharp. We attribute this to the induced chemical 1 physical interactions between the migrating ions and the external contacts. An initial high charge transfer resistance from the contact to the perovskite layer is reduced by surface reaction at higher bias, which is corroborated by impedance spectroscopy results (more discussion later). Above the SET voltage, the suppression of the charge transfer barrier from the electrode initiate the carrier conduction in the bulk, which is evident from the Ohmic regime (slope $\sim 1.2$, Fig. 1d) in the LRS. Subsequently, under negative applied bias, the device resistance changes from a LRS to the HRS by a "RESET" process to the non-conducting state with a current level of $10^{-8}-10^{-6} \mathrm{~A}$ (Fig. 1c). 

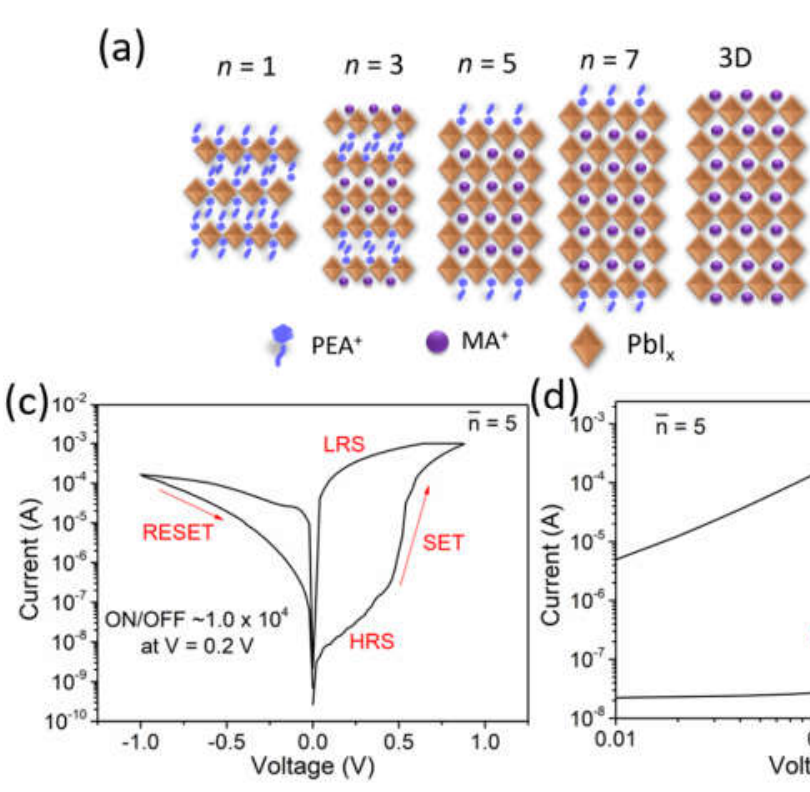

$\mathrm{Pbl}_{\mathrm{x}}$
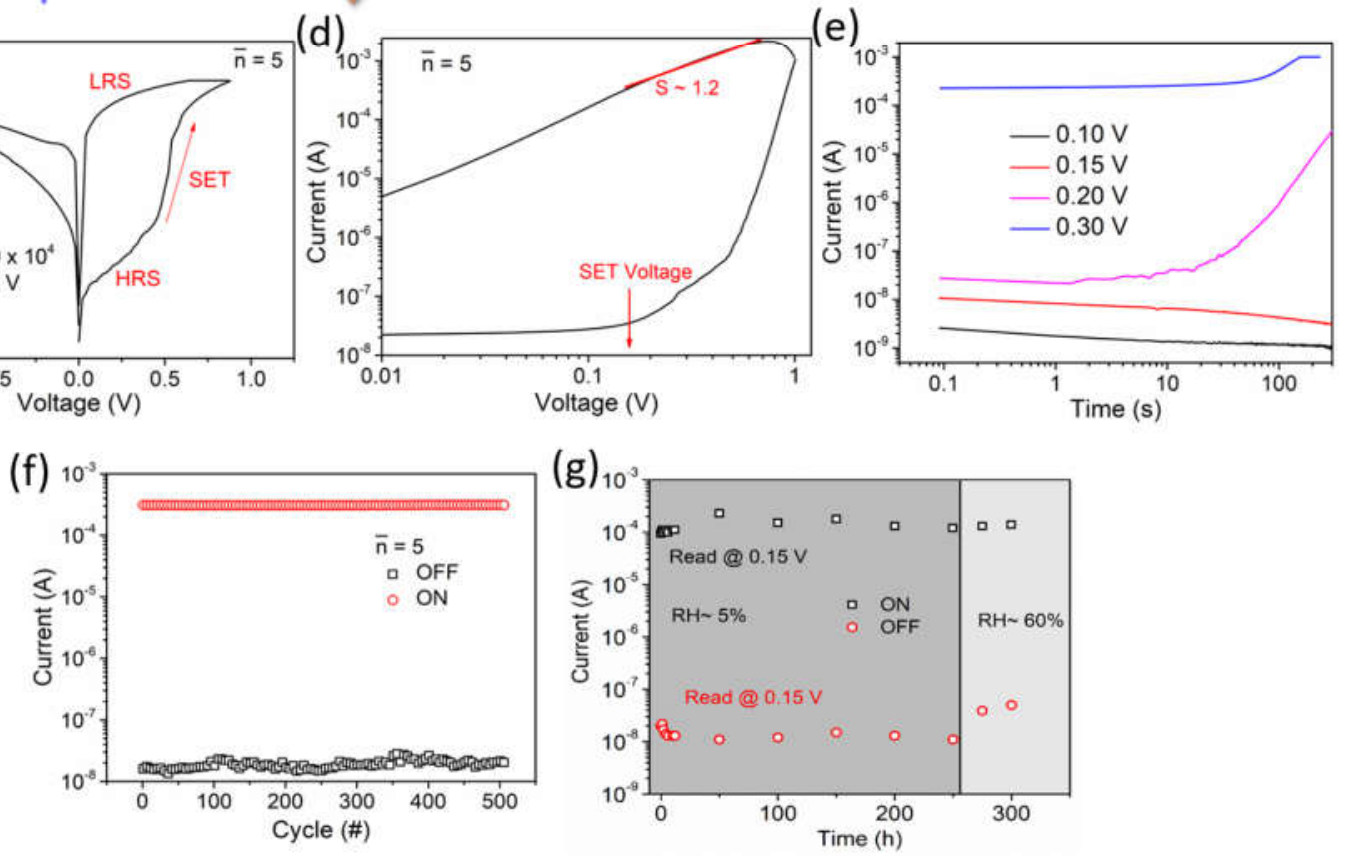

Fig. 1: Device electrical properties: (a) Crystal structure of ruddlesdsen-popper perovskite $(\bar{n}=1,3,5,7)$ and $3 \mathrm{D}$ perovskite, (b) schematic device structure of Glass/ITO/PEDOT:PSS/perovskite/PCBM/Ag. A thin layer of PCBM $(\sim 10 \mathrm{~nm})$, was inserted between the perovskite and top electrode to control Ag migration, (c) Semi-log I-V characteristics of $\bar{n}=5\left((\mathrm{PEA})_{2} \mathrm{MA}_{4} \mathrm{~Pb}_{5} \mathrm{I}_{16}\right)$ based ReRAM device with the scan rate of $\sim 100 \mathrm{mV} / \mathrm{s}$ (See Fig. 3a and Fig. S4 for others) - red arrows indicate the scan direction, (d) Log-log $I-V$ plot presenting the SET voltage in HRS and Ohmic conduction in LRS. (e) Transient current at different applied bias, (f) device endurance test for 500 cycles (applied voltage pulse $-1.5 /+1.5 \mathrm{~V}$ and read current at $0.15 \mathrm{~V})(\mathrm{g})$ data retention under $\sim 5 \%$ relative humidity (RH) (inside $\mathrm{N}_{2}$ glovebox) and ambient condition with $60 \% \mathrm{RH}$.

The selection of the optimum device operating conditions is essential because a higher applied bias can electrically short the device while a lower bias can lead to under-performance. From Fig. 1e, the OFF state remains with low current and nearly invariant at $<0.15 \mathrm{~V}$, while the current gradually increases at higher applied bias $(>0.15 \mathrm{~V})$ and finally switching to the $\mathrm{ON}$ state. The excellent switching stability of these devices (stable current measured at $0.15 \mathrm{~V}$ ) is confirmed by continuously triggering them from SET to RESET state by a voltage pulse slightly higher than the saturation voltage (i.e., $-1.5 /+1.5 \mathrm{~V}$ ) (Fig. 1f). Operational stability of our device is clearly demonstrated from the $500 I-V$ scans (Fig. S6). Strong data retention ability, a very important criterion for memory application, is measured for up to 250 hours (Fig.1g). However, the current in HRS increased gradually while exposed to the high humid ambient environment $(\sim 60 \% \mathrm{RH})$. The optical images of the device before and after two days exposure to the ambient 
(Fig. S7) confirm the degradation of $\mathrm{Ag}$, probably conversion into $\mathrm{AgO}_{\mathrm{x}}$, which could penetrate into the perovskite and react with it, thus reducing the device resistance in OFF state. ${ }^{25}$ However, the effect of ambient water cannot be excluded since it has been reported that formation of the water adduct with the perovskite sharply increases the conductivity. ${ }^{26}$

In perovskite ReRAM devices, two different mechanisms have been widely attributed to the SET and RESET processes: (1) Formation/rupture of the conducting filament from metal electrode and (2) Defects redistribution (predominantly iodide vacancies, iodine interstitials due to lower activation energy between $\sim 0.4-0.8 \mathrm{eV}$ for the migration) leading to the marked hysteresis. ${ }^{18,27}$ However, it is difficult to discriminate these mechanisms in a device solely based on the $I-V$ characteristics. In order to reveal or rule out the necessity of the metal filament formation for the switching mechanism, we carried out Scanning Kelvin probe microscopy (SKPM) and conductive atomic force microscopy (c-AFM) of bare perovskite films. Our results clearly show that switching occurs even without the top electrode present, therefore discounting the need for metal filament formation in the switching mechanism. A complete discussion can be found in the supporting information (Fig. S8). Based on these findings, we conclude that ionic redistribution can form the conducting channels for RS. ${ }^{22}$ However, it is important to note that such conducting pathways are transitory and will not form steady states as seen from the consistent drop in surface potential (Fig. S8d). Hence, ions redistribution alone for LRS/HRS will still not explain the long retention observed in Fig. 3g. An interplay between the perovskite and external contacts is at work in these ReRAM devices.
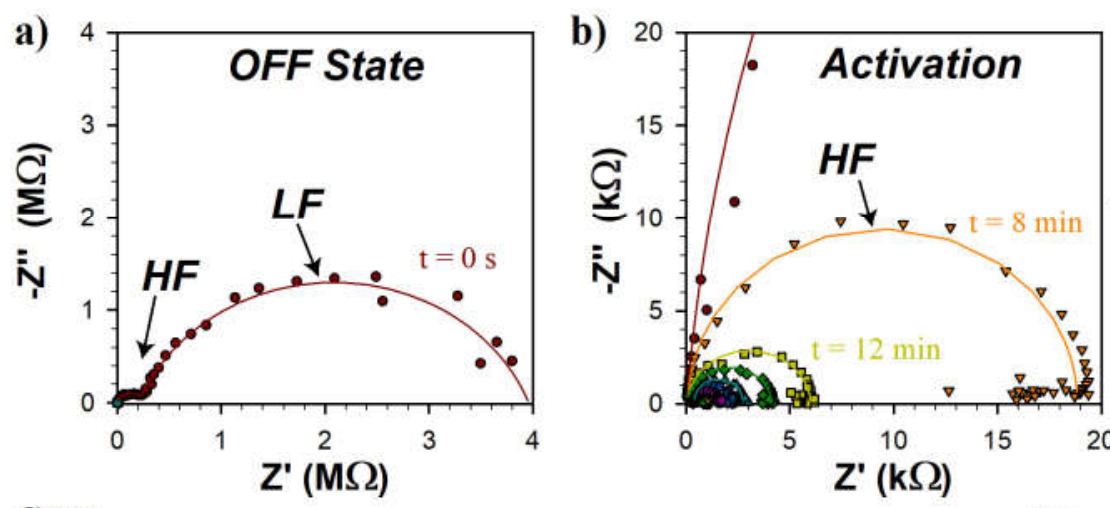

c)
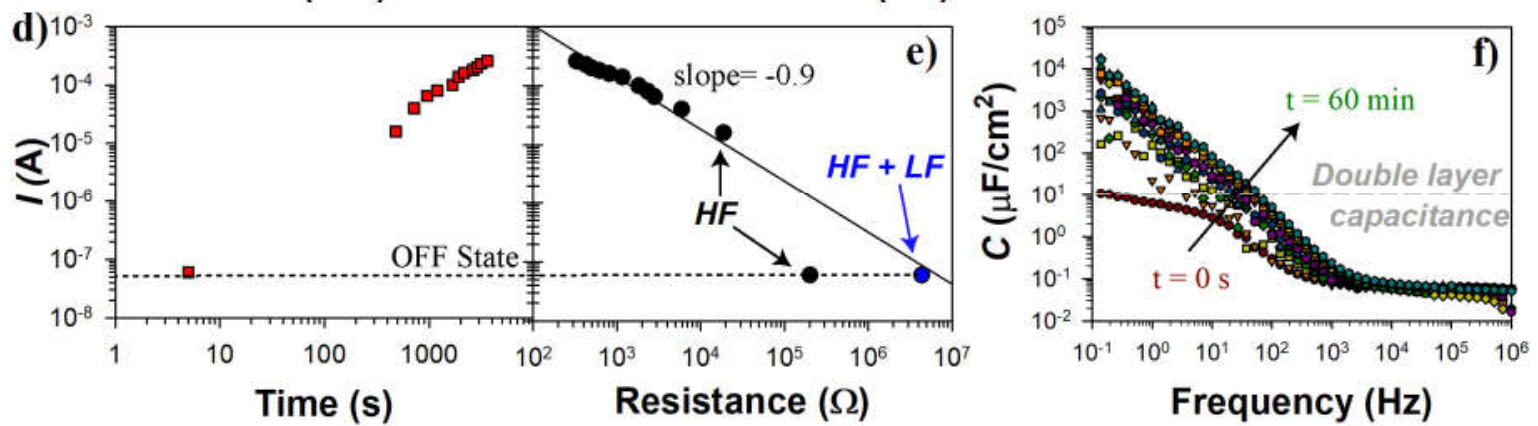

Fig. 2: Impedance Spectroscopy: a, b) Complex impedance spectra of a device polarized close to the activation voltage at $\mathrm{V}_{\mathrm{DC}}=0.2 \mathrm{~V}$ during IS measurements. IS frequency scans are repeated 15 times over one hour (i.e., 1 scan takes 4 minutes). The IS spectra repetition number is denoted by $\mathrm{t}=4 \times n$ minutes (where $n$ is an integer) in the graph (c) Equivalent circuit use to fit the impedance data (d) Current increases during repetition of the IS frequency scans 
and the device gets activated. (e) Fitting results of the resistance as a function of the current, (current and resistance errors are below $5 \%$ ). f) Capacitance frequency plot of the spectra shown in $a, b)$.

To investigate the origin of the strong and reversible switching between the LRS and HRS, we performed impedance spectroscopy, that allows the separation of different contributions of conduction by the coupling of resistances and capacitances. ${ }^{28}$ Here, the best device configuration (i.e., $\bar{n}=5$ ) is used. A DC bias slightly higher than the SET voltage switch to LRS is applied. We monitor the device behavior at $0.2 \mathrm{~V}$ by repetition of IS spectra measurement as the current gradually increases. The complex impedance plot is shown as a function of the time (IS scan number) in Figs. 2a,b for different range of impedance values. Fig. 2a show the initial HRS ( $t=$ 0 ) that corresponds to very high impedance. The occurrence of two arcs from ReRAM device is the typical response of perovskite devices and is usually described by the equivalent circuit shown in Fig. 2c. The high frequency (HF) arc contains information on the bulk conductivity of the perovskite film and the low frequency (LF) one relates to the perovskite/contacts interface (ion migration and accumulation). ${ }^{29}$ As shown in Fig. 2a, the HF arc is characterized by low impedance values of $0.2 \mathrm{M} \Omega$, associated to the bulk perovskite, while the LF arc provides large fitting values of $4.1 \mathrm{M} \Omega$, which is related to the resistive elements at the perovskite/contact interface. Remarkably, as the device is activated, the LF arc disappears (i.e., only HF arcs remains in Fig. 2b). The current abruptly increases 2 orders of magnitude (Fig. 2d, time $=480 \mathrm{~s}$, 8 minutes). Results show that the bulk-conduction (HF) remains virtually unchanged, while the dramatic change in charge injection characteristics at the interface leads to the absence of the large LF arc seen in Fig. 2a. Instead of the LF arc, some inductive effects in the low-frequency range are observed, correlated with electrochemical reactions and corrosion in several materials in battery configuration. ${ }^{30,31}$ By repetition of the IS measurements above SET voltage, there is a rise of the current and the HF resistance decreases with time (Fig. 2d), however, the inductive effect is always present. Fig. 2e explicitly demonstrates a linear correlation between the sum of all resistances ( $R_{\text {series, }}, R_{H F}$ and $R_{L F}$ ) observed in the IS measurements and the injected current. The slope is confirmed to be -0.9 , indicating an Ohmic response when all the resistances are taken into account, obeying Ohm's law.

Additional information about charge distribution in the device is obtained by plotting the capacitance-frequency plot (Fig. 2f) where charge accumulation at the perovskite/external contacts interface is related to the formation of an ionic double layer connected to the LF arc. ${ }^{32,33}$ In perovskites, double layer capacitance arises from the accumulation of iodine ions/vacancies at the electrode with typical values in the tens of $\mu \mathrm{F} / \mathrm{cm}^{-2}$. These values are only detected in the OFF state where the LF signal connects the presence of ions at the contacts $\left(\mathrm{C}_{\mathrm{DL}} \sim \mu \mathrm{F} / \mathrm{cm}^{-2}\right)$ with impeded charge carrier injection from the contacts $\left(\mathrm{R}_{\mathrm{LF}} \sim \mathrm{M} \Omega\right)$. Thereafter, when the device approaches the SET process, the capacitance increases dramatically up to values of $10 \mathrm{mF} / \mathrm{cm}^{2}$ at frequencies of $10 \mathrm{mHz}$, more than three orders of magnitude, higher than typical double layer and Helmholtz layer capacitances. With common capacitance values of conversion reaction in batteries the capacitance results points to a chemical reaction of iodide ions with the external Ag interfaces that lead to formation of AgI. ${ }^{34}$ As the SET process activates, the LF arc disappears with subsequent formation of inductive features, which have been previously correlated with the reaction at electrode/active layer interface, resulting in the lower series resistance of the device.

Next, $I-V$ characteristics in different ReRAM devices are systematically investigated to uncover the interplay between the perovskite and external contacts. We fabricated a whole series of 
devices containing perovskites with different $\bar{n}$ values $(\bar{n}=1,3,5,7, \infty / 3 \mathrm{D})$ with contacts modification. Only, selected $I-V$ curves are shown here for clarity and others are presented as supporting information (Fig. S4, 10, 11). Based on the $I-V$ characteristics (Fig. 3a), the OFFstate current clearly depends on the perovskite composition (i.e., $\bar{n}$ values). In RPP thin films, $\mathrm{PbI}_{6}$ octahedral layers are the predominant conducting channels, therefore overall bulk conductivity in thin film depends on the number of layers. Between two electrodes, these layers are (i) un-interruptedly aligned in (110) plane parallel to each other from the bottom to top electrode; and/or (ii) partially cross-linked; (iii) higher $\bar{n}$ value layers are connected via lower $\bar{n}$ values layers. ${ }^{35,36}$ In either case, charge transport across the perovskite layer is affected by PEA layers, which have relatively poor charge carrier mobility to control the OFF state. The balance in the composition of 3D octahedral and PEA layers and their well distribution in $\bar{n}<5$ leads to very high resistance in the OFF state. On the other hand, in higher order layered perovskites $(\bar{n}>$ 5 ), the dominant presence of the 3D layers leads to well-connected channels across the electrodes and charge transport dominant though these channels and off state resistance is reduced to show a relatively higher current. All these devices at low voltage $(<0.4 \mathrm{~V})$ are in high resistance state (HRS or OFF state i.e. $>10^{5} \Omega$ ) with measured low level of current. The SET voltage is approximately $0.4,0.3,0.2,0.22,0.2 \mathrm{~V}$ for $\bar{n}=1,3,5,7$ and 3D based ReRAM devices, respectively (Fig. 3b). The SET voltage of these devices are estimated from the deviation of the current from slope 1 in HRS (Fig. 1e). ${ }^{18}$ This trend is in agreement with the enhanced ionic transport observed for the 3D perovskites compared to 2D and mixed perovskites. ${ }^{37}$ Therefore, we establish a connection between ionic migration and SET voltages in ReRAM configuration as discussed below. The maximum ON/OFF ratio $\sim 1 \times 10^{4}$ and minimum value of SET voltage in $\bar{n}=5$ indicates that RS is most efficient as compared to other layered perovskites (Fig. $\mathbf{3 b}$ ). The variation of the ON/OFF ratio for different $\bar{n}$ value RP perovskites with similar thickness ( $270 \pm 20 \mathrm{~nm}$ ) is also a secondary evidence to confirm that RS in layered RPP is not caused by metal filament formation. Other than the $\bar{n}$ values, the electronic conductivity in RPP or OFF state current is also determined by the terminating end of perovskite structure and the interaction at perovskite/contact interface, which control the charge transfer to the conduction band. For example, the presence of iodine or MA vacancies $\left(V_{I}\right.$ and $\left.V_{M A}\right)$ at $\mathrm{CH}_{3} \mathrm{NH}_{3} \mathrm{PbI}_{3} / \mathrm{TiO}_{2}$ interface modify the energy landscape and hinder the charge extraction. ${ }^{38}$ Similarly, a high local concentration of terminal iodine ions in the presence of oxidative conditions (high hole concentration at the $\mathrm{Ag}$ contact), form metal-halides (i.e., $\mathrm{AgI}$ or $\mathrm{AuI}_{3}$ ) bonds locks the iodine at the contact and modifies the injection properties of the contact reducing the charge transfer resistance. ${ }^{39}$

Several top electrodes ( $\mathrm{Ag}, \mathrm{Au}, \mathrm{ZnO} / \mathrm{Ag}, \mathrm{MoO}_{3} / \mathrm{Ag}, \mathrm{Al} \ldots$ ) and bottom electrodes (ITO, Pt, PEDOT:PSS) have been used in the literature in combination with either iodide or bromide based perovskites. Whilst some electrode/perovskite combinations provide better RS than others, a complete picture of the most suitable combination is still lacking. To uncover the mechanism, various device configurations with different perovskite composition and external contacts (Fig. 3c, 3d and Fig. S10, 11) were fabricated and their $I-V$ characteristics are investigated. The lack of current switching in devices with top bare $\mathrm{Au}$, thin PCBM/Au and with thick PCBM/Ag highlights the significance of the population of migrating ions (relatively less migrating ions in $\bar{n}$ $=1$ compared to $\bar{n}=5$ ) and their reactivity with the electrodes (higher reactivity of Ag compared to $\mathrm{Au}$ ). Here we propose that the unfavorable chemical reactivity of $\mathrm{Au}$ with $\mathrm{I}^{-}$requires higher 
concentration of ions, provided by the 3D perovskite, and direct contact with perovskite are needed for the RS. Therefore, we show that the ion's population, their migration and reactivity with the contacts is essential to achieve the memory effect.

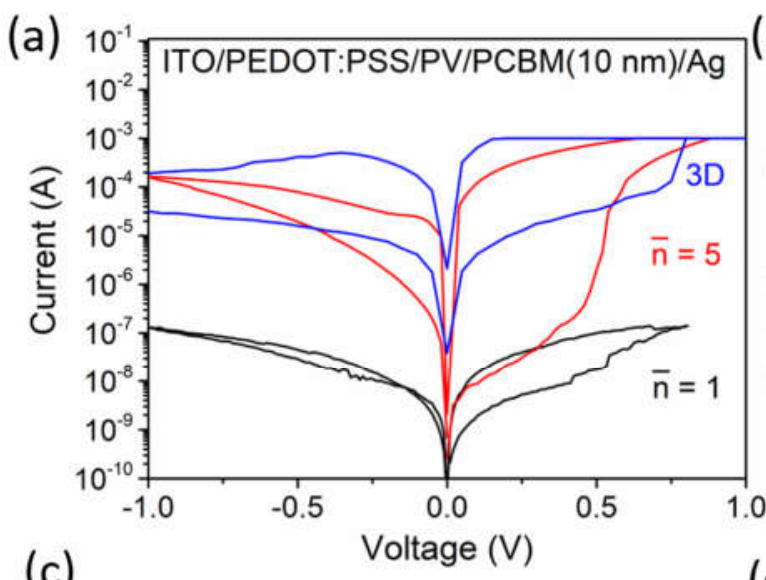

(c)
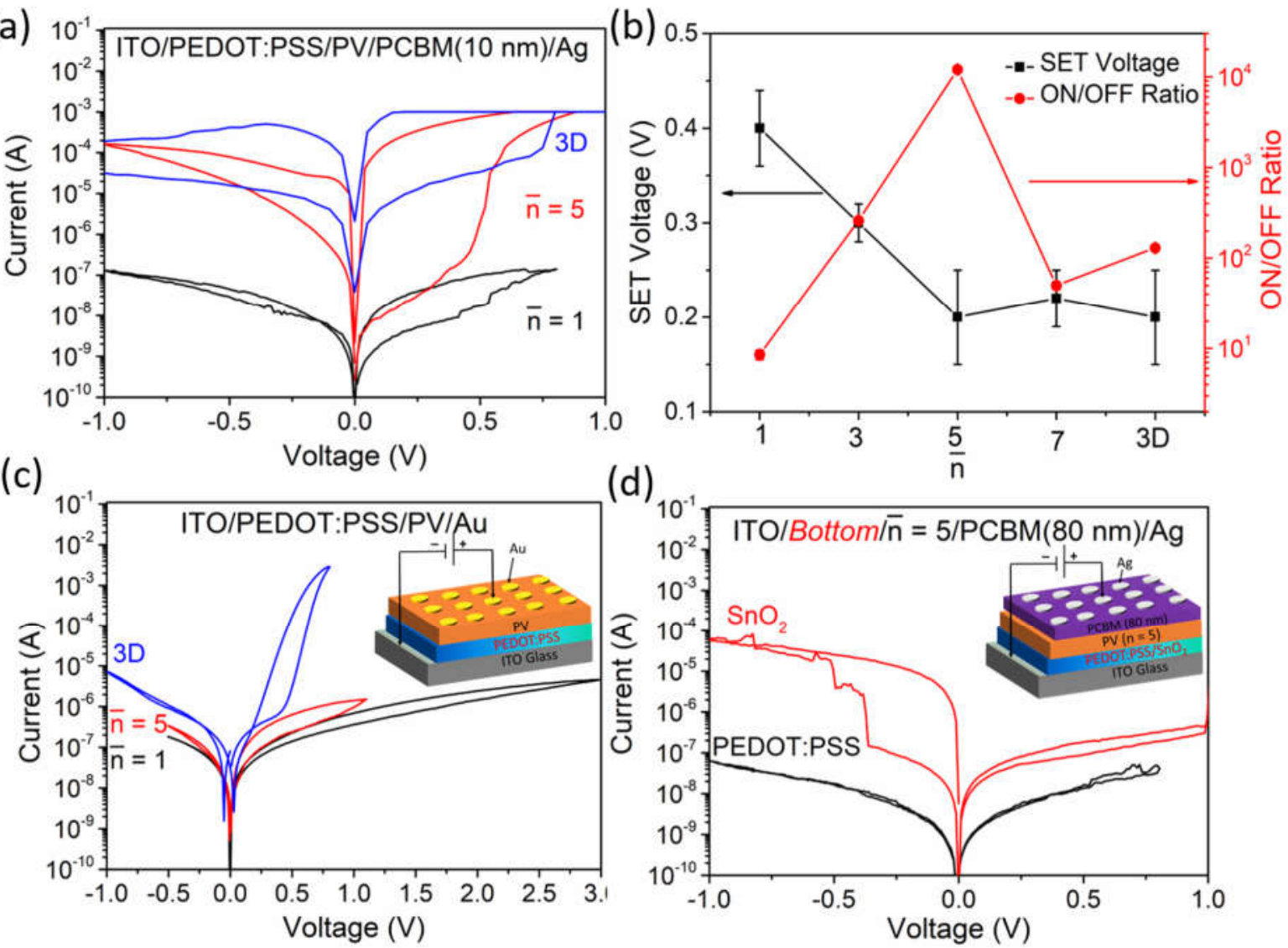

(d)

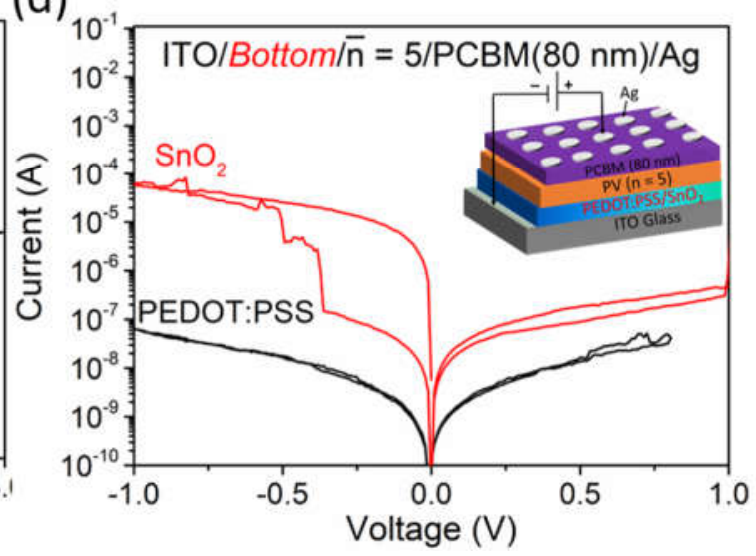

Fig. 3: Electrical properties for different ReRAM devices: (a) $I-V$ curves for devices containing perovskites with different $\bar{n}$ indices $(\bar{n}=1,5, \infty / 3 \mathrm{D})$ in a ITO/PEDOT:PSS/Perovskite/PCBM/Ag configuration (see Fig. S9 for $\bar{n}=3$ and 7). (b) Summary of SET voltages and ON/OFF ratios for different devices. (c) $I-V$ curve for $\bar{n}=1,5$, $\infty / 3 \mathrm{D}$ based devices containing bare Au top electrode in the configuration glass/ITO/PEDOT:PSS/PV/Au (d) $I-V$ curve for devices containing different bottom electrodes and a thick layer of PCBM ( $80 \pm 10 \mathrm{~nm})$ between perovskite $(\bar{n}=5)$ and $\mathrm{Ag}$.

Lastly, we are able to tune the current switching quadrant by controlling the chemical/physical reaction of migrating ions at perovskite/contact interfaces, thus verifying our findings. The introduction of thick PCBM layer $(80 \pm 10 \mathrm{~nm})$ in our best device creates a more robust physical barrier that prevents the interaction between I- and Ag to form AgI. Hence, the absence of RS in these devices (Fig. 3c). On the other hand, the non-interactive nature of PEDOT:PSS with migrating ions does not contribute to the charge transfer and $\mathrm{ON}$ state retention and therefore no current switching at negative applied bias (note the highly symmetric curve). Alternatively, if the PEDOT:PSS layer is replaced by $\mathrm{SnO}_{2}$ whilst keeping the thick PCBM layer, the interactions of $\mathrm{SnO}_{2}$ with migrating ions lead to a switching behavior at negative bias (Fig. 3d). This is probably due to $\mathrm{I}^{-}$ions coordinating with $\mathrm{Sn}^{3+}$ defects present in $\mathrm{SnO}_{2}$ that yields the RS. Overall, we conclude that both electrode materials $\left(\mathrm{Ag}\right.$ and $\left.\mathrm{SnO}_{2}\right)$ may lead to chemical 
interactions with migrating ions that can be tuned to prepare ReRAM devices. Alternatively, PEDOT:PSS and PCBM offer a physical barrier to migrating iodine ions and do not provide strong interactions, making the layers inert to ReRAM response. Selection of materials can be based on these simple design rules to build up a complete library.

Based on the understanding from a combination of impedance spectroscopy and the results of different configurations, we propose a general switching mechanism that relies on the interplay between two kinetics; (i) ion migration in the perovskite and (ii) reversible chemical interactions with the external contacts. This mechanism relies on the current understanding of the kinetic components of the impedance response that increases the capacitance in a phenomenon related to the LF inductive effect and the chemical reactivity of migrating ions with external contacts. ${ }^{40}$ Fig. 4 represents the best performing configuration described in this work. Here, the reversible reaction of formation and dissolution of a AgI monolayer at the interface is suffice to modify the injection properties to control the charge transfer. Therefore, the selection of contacts and their redox potentials are the primary factors to determine the chemical reaction at the interface and thus the required onset potential to SET process to observe the memory effect. Similarly, the high degree of the reversibility of the reaction is imperative for high ON/OFF ratio in ReRAM device.
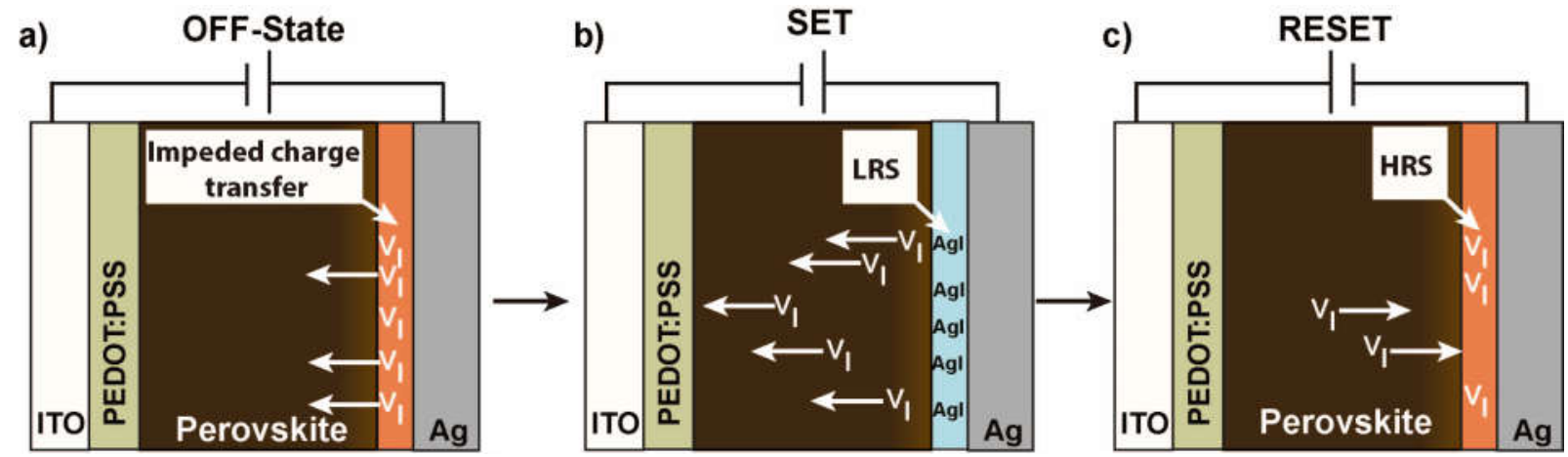

Fig. 4: Proposed switching mechanism: (a) OFF State: Iodine vacancies $\left(V_{I}\right)$ termination at the interface limit the charge transfer from Ag to the perovskite layer, (b) SET process: under an applied electrical the perovskite termination is modified by $V_{I}$ migration to form a thin layer of AgI, beneficial for the efficient charge transfer to form LRS. (c) RESET process: at the reverse polarity of the applied bias, $V_{I}$ migrates to the opposite direction and end up pilling at the electrode leading to the HRS. These processes are reversible based on the reaction at the contact interfaces.

\section{Conclusions}

Here, we demonstrated and investigated the RS in RP perovskite using different ReRAM device configurations. Optimal $\bar{n}=5$ composition shows the best performance with minimal SET operative voltage and highest ON/OFF ratio. The reliable endurance for 500 cycles and long data retention for 250 hours in an inert environment with subsequent 50-hour data retention under $60 \% \mathrm{RH}$ shows the excellent potential of RP perovskites for resistive memory applications. Microscopic studies reveal the surface conductivity switching in the absence of metal filament highlight the importance of the chemical/physical reaction at the interface to control the RS. We propose a general method to tune/enhance memory effect ReRAMs in the desired $I-V$ quadrant by selection of appropriate RP perovskite and electrode materials. Our findings uncover new 
opportunities offered by RP perovskites for RS and reliable data retention for memory device applications. Importantly, these findings may help illuminate the connections between ion migration and defects formation that give rise to the hysteresis effects plaguing perovskite optoelectronics. 


\section{References}

1. A. Kojima, K. Teshima, Y. Shirai and T. Miyasaka, Journal of the American Chemical Society 131 (17), 6050-6051 (2009).

2. W. Xu, Q. Hu, S. Bai, C. Bao, Y. Miao, Z. Yuan, T. Borzda, A. J. Barker, E. Tyukalova, Z. Hu, M. Kawecki, H. Wang, Z. Yan, X. Liu, X. Shi, K. Uvdal, M. Fahlman, W. Zhang, M. Duchamp, J.-M. Liu, A. Petrozza, J. Wang, L.-M. Liu, W. Huang and F. Gao, Nature Photonics (2019).

3. H. J. Snaith, A. Abate, J. M. Ball, G. E. Eperon, T. Leijtens, N. K. Noel, S. D. Stranks, J. T.-W. Wang, K. Wojciechowski and W. Zhang, The Journal of Physical Chemistry Letters 5 (9), 1511-1515 (2014).

4. F. Ebadi, N. Taghavinia, R. Mohammadpour, A. Hagfeldt and W. Tress, Nature Communications 10 (1), 1574 (2019).

5. H. Kim, L. Zhao, J. S. Price, A. J. Grede, K. Roh, A. N. Brigeman, M. Lopez, B. P. Rand and N. C. Giebink, Nature Communications 9 (1), 4893 (2018).

6. H. Cho, C. Wolf, J. S. Kim, H. J. Yun, J. S. Bae, H. Kim, J.-M. Heo, S. Ahn and T.-W. Lee, Advanced Materials 29 (31), 1700579 (2017).

7. J.-W. Lee, S.-G. Kim, J.-M. Yang, Y. Yang and N.-G. Park, APL Materials 7 (4), 041111 (2019).

8. R. Gottesman, P. Lopez-Varo, L. Gouda, Juan A. Jimenez-Tejada, J. Hu, S. Tirosh, A. Zaban and J. Bisquert, Chem 1 (5), 776-789 (2016).

9. T. Zhang, X. Meng, Y. Bai, S. Xiao, C. Hu, Y. Yang, H. Chen and S. Yang, Journal of Materials Chemistry A 5 (3), 1103-1111 (2017).

10. A. Guerrero, J. You, C. Aranda, Y. S. Kang, G. Garcia-Belmonte, H. Zhou, J. Bisquert and Y. Yang, ACS Nano 10 (1), 218-224 (2016).

11. K. Domanski, B. Roose, T. Matsui, M. Saliba, S.-H. Turren-Cruz, J.-P. Correa-Baena, C. R. Carmona, G. Richardson, J. M. Foster, F. De Angelis, J. M. Ball, A. Petrozza, N. Mine, M. K. Nazeeruddin, W. Tress, M. Grätzel, U. Steiner, A. Hagfeldt and A. Abate, Energy \& Environmental Science 10 (2), 604-613 (2017).

12. G. Lin, Y. Lin, R. Cui, H. Huang, X. Guo, C. Li, J. Dong, X. Guo and B. Sun, Journal of Materials Chemistry C 3 (41), 10793-10798 (2015).

13. Z. Xiao and J. Huang, Advanced Electronic Materials 2 (7), 1600100 (2016).

14. S.-I. Kim, Y. Lee, M.-H. Park, G.-T. Go, Y.-H. Kim, W. Xu, H.-D. Lee, H. Kim, D.-G. Seo, W. Lee and T.-W. Lee, Advanced Electronic Materials 0 (0), 1900008.

15. Y. Wang, Z. Lv, L. Zhou, X. Chen, J. Chen, Y. Zhou, V. A. L. Roy and S.-T. Han, Journal of Materials Chemistry C 6 (7), 1600-1617 (2018).

16. E. J. Yoo, M. Lyu, J.-H. Yun, C. J. Kang, Y. J. Choi and L. Wang, Adv. Mater. 27 (40), 6170-6175 (2015).

17. H. Ma, W. Wang, H. Xu, Z. Wang, Y. Tao, P. Chen, W. Liu, X. Zhang, J. Ma and Y. Liu, ACS Applied Materials \& Interfaces 10 (25), 21755-21763 (2018).

18. C. Gu and J.-S. Lee, ACS Nano 10 (5), 5413-5418 (2016).

19. P. Calado, A. M. Telford, D. Bryant, X. Li, J. Nelson, B. C. O'Regan and P. R. F. Barnes, Nature Communications 7, 13831 (2016).

20. J. S. Han, Q. V. Le, J. Choi, K. Hong, C. W. Moon, T. L. Kim, H. Kim, S. Y. Kim and H. W. Jang, Advanced Functional Materials 28 (5), 1705783 (2018).

21. X. Zhu, J. Lee and W. D. Lu, Advanced Materials 29 (29), 1700527 (2017).

22. X. Guan, W. Hu, M. A. Haque, N. Wei, Z. Liu, A. Chen and T. Wu, Advanced Functional Materials 28 (3), 1704665 (2018).

23. J.-Y. Seo, J. Choi, H.-S. Kim, J. Kim, J.-M. Yang, C. Cuhadar, J. S. Han, S.-J. Kim, D. Lee, H. W. Jang and N.-G. Park, Nanoscale 9 (40), 15278-15285 (2017).

24. S. Ge, Y. Huang, X. Chen, X. Zhang, Z. Xiang, R. Zhang, W. Li and Y. Cui, Advanced Materials Interfaces 6 (7), 1802071 (2019).

25. J. Li, Q. Dong, N. Li and L. Wang, Advanced Energy Materials 7 (14), 1602922 (2017). 
26. C. Aranda, C. Cristobal, L. Shooshtari, C. Li, S. Huettner and A. Guerrero, Sustainable Energy \& Fuels 1, 540 - 547 (2017).

27. J. Choi, S. Park, J. Lee, K. Hong, D.-H. Kim, C. W. Moon, G. D. Park, J. Suh, J. Hwang, S. Y. Kim, H. S. Jung, N.-G. Park, S. Han, K. T. Nam and H. W. Jang, Adv. Mater. 28 (31), 6562-6567 (2016).

28. A. Guerrero, G. Garcia-Belmonte, I. Mora-Sero, J. Bisquert, Y. S. Kang, T. J. Jacobsson, J.-P.

Correa-Baena and A. Hagfeldt, J. Phys. Chem. C 120 (15), 8023-8032 (2016).

29. W. Peng, C. Aranda, O. M. Bakr, G. Garcia-Belmonte, J. Bisquert and A. Guerrero, ACS Energy Lett. 3 (7), 1477-1481 (2018).

30. L. C. Bai, B. E. ;, Journal Electrochemical Society 138 (10), 2897-2907 (1991).

31. R. F. A. P. Jargelius-Pettersson, B. G.;, J. Electrochem. Soc. 145 (5), 1462-1469 (1998).

32. O. Almora, A. Guerrero and G. Garcia-Belmonte, Appl. Phys. Lett. 108 (4), 043903 (2016).

33. O. Almora, I. Zarazua, E. Mas-Marza, I. Mora-Sero, J. Bisquert and G. Garcia-Belmonte, J. Phys. Chem. Lett. 6 (9), 1645-1652 (2015).

34. F. Martinez-Julian, A. Guerrero, M. Haro, J. Bisquert, D. Bresser, E. Paillard, S. Passerini and G. Garcia-Belmonte, The Journal of Physical Chemistry C 118 (12), 6069-6076 (2014).

35. H. Tsai, R. Asadpour, J.-C. Blancon, C. C. Stoumpos, J. Even, P. M. Ajayan, M. G. Kanatzidis, M. A. Alam, A. D. Mohite and W. Nie, Nature Communications 9 (1), 2130 (2018).

36. A. Krishna, S. Gottis, M. K. Nazeeruddin and F. Sauvage, Advanced Functional Materials 29 (8), 1806482 (2019).

37. C. Li, A. Guerrero, S. Huettner and J. Bisquert, Nature Communications 9 (1), 5113 (2018).

38. J. M. Azpiroz, E. Mosconi, J. Bisquert and F. De Angelis, Energy Environ. Sci. 8, 2118--2127

(2015).

39. J. Pospisil, A. Guerrero, O. Zmeskal, M. Weiter, J. J. Gallardo, J. Navas and G. Garcia-Belmonte, Advanced Functional Materials 0 (0), 1900881.

40. H. Wang, A. Guerrero, A. Bou, A. M. Al-Mayouf and J. Bisquert, Energy \& Environmental Science 12 (7), 2054-2079 (2019).

\section{Acknowledgments}

Financial support from the JSPS-NTU Joint Research Project M4082176; the Ministry of Education AcRF Tier 1 grant RG173/16 and Tier 2 grants MOE2015-T2-2-015 and MOE2016-T2-1-034, and MOE2017-T2-1-110; and from the Singapore National Research Foundation through the NRF Investigatorship Programme NRF-NRFI-2018-04 are gratefully acknowledged. We also thank the financial support from the Ministerio de Ciencia, Innovación y Universidades of Spain under project (MAT2016-76892-C3-1-R). A.G. would like to thank MICINN for a Ramón y Cajal Fellowship (RYC-201416809). University Jaume I is also acknowledged for financial support (UJI-B2017-32).

\section{Authors contribution}

A.S. and T.C.S. conceived the idea for the manuscript. A.S. prepared thin films and devices and conducted electrical, optical and structural characterizations. A.G. performed all impedance spectroscopy measurements and analysed the data with J.B. Q.Z. conducted morphological and conducting-AFM characterizations. T.C.S., J.B., A.S. A.G. and Q.Z. analyzed the data and wrote the manuscript.

Competing interests: The authors declare there is no competing interests. 